\title{
LENGTH OF TIME INTERVAL IN SUCCESSIVE ASSOCIATION
}

\author{
BY HARVEY CARR \\ The Univer sity of Chicago
}

This paper presents data obtained by the use of animals in the alternation problem. Two diverging paths $R$ and $L$ were presented and the animals were required to learn to choose between them in the given temporal sequence of $R, L, R, L$, $R, L$, etc., for each day's series of trials. The mastery of such a sequence of choices involves the formation of an association between each of the two responses and a certain stimulus. Naturally the two stimuli must alternate in the given sequence. Conditions were arranged so that presumably the animal would be compelled to utilize for each choice the somatic (cutaneous, kinæsthetic, and organic) stimuli resulting from the preceding run in order to master the problem. The problem thus involves the formation of an association between two successively presented terms, each response and the sensory resultants of the preceding response. The resulting coördination may be regarded as somewhat similar to the act of walking, in which the señsations from each step constitute in part the stimulus for the succeeding step. The time allowed for eating between runs was varied for the different groups of animals. In this way the length of the interval between the two terms to be associated was varied, and our problem may be stated as the influence of the length of the time interval between two successively presented terms upon the speed of their association.

A plan of the problem box employed in the experiment is represented in Figure r. Its dimensions are $23 / 4 \times 5 \mathrm{ft}$., with $4 \times 4$ in. runways and a $10 \times 13$ in. food box. With the exception of the food box $A$, the apparatus was covered with a glass top. An initial runway $C$ opens from the food box by the opening $B$ which can be closed by a sliding door. At 
the end of this runway are two diverging paths $R$ and $L$ which finally merge into a common path $D$ which in turn leads to the food box by the door $E$. These return paths to the food can be blocked as desired by

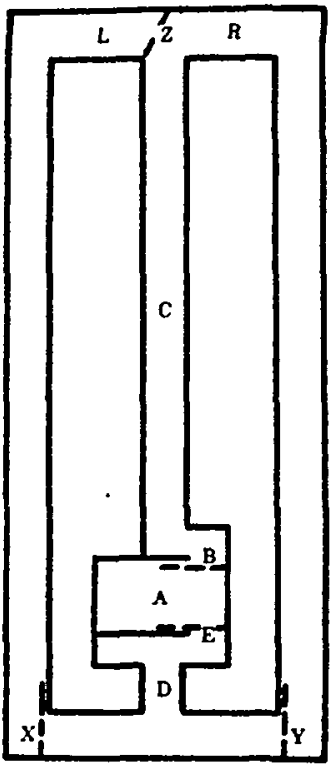

Frgure x. Problem Box. the sliding doors $X$ and $Y$.

The rats were required to master the habit of alternating their choices between the two diverging paths in the definite sequence of $R, L, R, L, R, L$, etc., for each day's series of trials. The animal was placed in the food box and forced through the door $B$ which was then closed. In the first trial of each day's series of runs, the doors $X$ and $Y$ were arranged so that food could be obtained only by means of path $R$. In the succeeding trial the doors were rearranged so that path $L$ was open and $R$ closed. Between the successive runs for a day's series, the rats were allowed to eat for a definite time interval. This interval was measured by means of a metronome adjusted to ring a bell every five seconds. The progress of the animals in mastering the association was measured by the percentage of successful choices in each successive 100 trials. Naturally the initial records will approximate 50 per cent., and the values will increase to 100 per cent. as the problem is mastered.

The sensory basis of the two responses must consist of two different stimuli which alternate in the given sequence. There are several stimuli which fulfill these conditions:

I. The responses may be based upon stimuli involved in the adjustment of the doors $X$ and $Y$. This possibility was eliminated by control tests in which the adjustments were made after the animals had made their choice, or in which both doors were left open for a number of consecutive trials. The utilization of such stimuli would not, however, invalidate any conclusions concerning the relation of the time interval 
to the rate of learning. It was the general practice throughout the experiment to adjust these doors immediately after the animal had entered the food box. As a consequence the two terms to be associated would be separated by the interval of time allowed for eating between runs.

2. The animals may have been guided by two different modes of handling while being inserted through the door $B$. To avoid this possibility the manner of handling and placing the rats in the initial runway was varied during the mastery of the problem and in control tests at the termination of the experiment.

3. The rats may have responded visually to two distinctive attitudes assumed by the experimenter while they were traversing runway $C$. It was soon observed that the experimenter naturally tended to act differently as the sequence of correct choices was alternated. The experimenter attempted to inhibit this tendency and maintain a constant position and attitude for all trials until the choice had been effected. The writer attempted to influence the choices of the rats by such means in one test. The orientation of the animal in relation to the experimenter while traversing the initial runway is such that the utilization of visual stimuli from the experimenter would presumably be difficult. The possibility was effectually eliminated in one experiment by a control test in which the initial runway was entirely covered so that visual stimuli from the experimenter were excluded until after the animals had entered one of the return paths.

4. Smell is also a possibility. One may assume that the odor left from the last run is stronger than that resulting from the run preceding it. Choice would thus be based upon a difference of olfactory strength of the two trails. Mastery of the problem would involve the selection of the weaker of the two olfactory stimuli. The pathways were thoroughly washed every two or three weeks during the course of the experimentation and this procedure disturbed in no way the ability to make successful choices on the succeeding day. If olfactory stimuli were utilized, choice would presumably 
be impossible until the animal had reached the point of divergence at the end of alley $C$. As a matter of fact the selection of a path in the majority of cases had been determined before this point was reached. This fact was quite evident from the behavior of the animals and the phenomenon was noted and commented upon by several observers. Before reaching the end of the initial pathway, the animals generally manifested a bodily attitude adaptive and preparatory to the turn involved in entering the selected return path. In case of a leftward turn, the animals veered over to the left side of the alley with the body twisted and curved in that direction. It was quite evident that the whole organism was being adjusted for that sort of a turn, and in the majority of cases in the later stages of the experiment the observer could easily predict the path to be selected when the animal was from one to two feet away. A few rats at certain stages in the mastery of the problem apparently did not make their choice until the two diverging paths were reached; they ran the initial path in a desultory fashion and then exhibited hesitation and wavering between the two possibilities before a final selection was made. This type of behavior was the exception however.

5. Another possibility was suggested by Mr. E. S. Robinson. The hypothesis assumes that the rat first develops a tendency to repeat the previous choice. The effect of each run is retained during the interval and the tendency is awakened by the stimulus from the apparatus when the rat is inserted into the entrance alley. Such a tendency toward repetition is involved in the formation of position habits. Under our conditions this tendency to repeat the previous act will naturally result in an error or wrong choice. The rat now learns to inhibit this wrong response and choose the opposite path. The rat starts to turn in the wrong direction and this sensory attitude becomes the stimulus to the correct choice. On this hypothesis the production and elimination of errors are necessary stages in the mastery of the problem. Furthermore the two associated factors, the correct response and the somatic stimuli involved in the 
tendency to enter the wrong path, are presented in immediate succession. The behavior described in the previous section would seem to eliminate this possibility. The assumed process of gradually inhibiting the wrong act and substituting the correct response in its place was not observed. Neither did the animals when placed in the initial runway first manifest an attitude antagonistic to their subsequent choice and then reverse it. The only observable attitude was one adapted to the succeeding choice.

6. The animal may assume and maintain two distinctive orientations in the food box during the interval allowed for eating. We were unable to observe such attitudes, however. On the contrary, each rat assumed a characteristic attitude which was constant for the majority of the trials. The construction of the apparatus minimizes this possibility inasmuch as both return routes finally merge into a common path and the animals are compelled to approach the food in an identical manner irrespective of which return route was chosen.

7. The remaining factor is the somatic sensitivity involved in the preceding run. The two responses necessarily entail two distinctive sets of somatic stimuli, and each of these may well constitute the basis of the subsequent choice. The fact that choice was apparently determined by an organic set manifested while the rat was in the initial runway is readily explicable on this hypothesis. Proof of the efficacy of this factor is primarily a matter of exclusion, the elimination of all other possibilities.

I. The first experiment was conducted by a graduate student, Miss Louisa Lewis. Three groups of eight rats each were employed, but several were eliminated by various causes during the experimentation. Complete records were obtained from five rats of group $A$, three of group $B$ and seven of group $C$. The time intervals for groups $A, B$, and $C$ were 15, 25, and 35 seconds respectively. Similar conditions obtained for the three groups throughout. Twenty trials per day were given each animal with occasional exceptions when the condition of the animals did not warrant that number. 
The animals were tested daily with the exception of Sunday. The tests were omitted for a period of ten days at the end of I,300 trials. To facilitate the progress of the experiment, two animals were generally tested simultaneously; one rat was allowed to run while the other was engaged in eating during the specified time interval. The experiment was continued until each rat attained a record of 90 per cent. of correct choices for 100 trials.

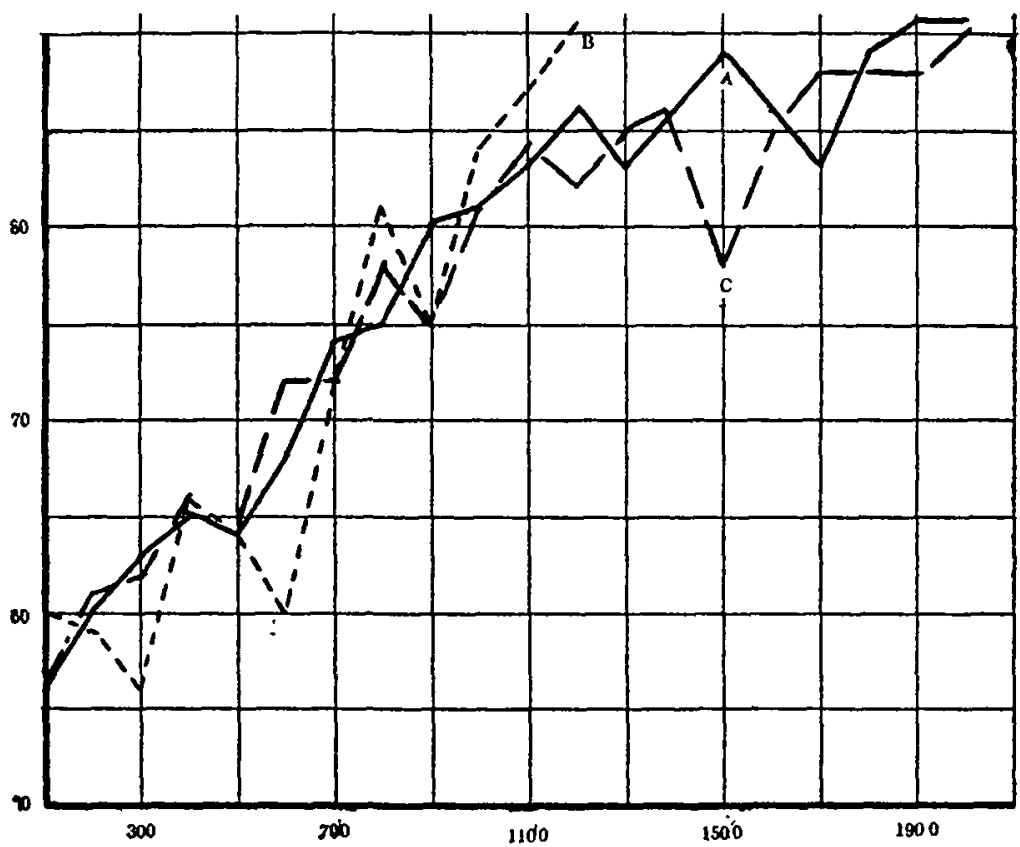

Figure 2. The graphs $A, B$, and $C$ represent the average percentage of correct choices for successive 100 trials for the intervals of 15,25 , and 35 seconds respectively.

Graphs $A, B$ and $C$ of Figure 2 represent the records of the three groups. The values represented are the average number of correct choices per group for each successive 100 trials. All animals succeeded in attaining a record of 90 per cent. of correct choices for 100 trials. The three groups were subjected to the various control tests enumerated above with the exception of that for visual cues from the experimenter while traversing the initial path. 
Several significant features of the results deserve comment: (I) All curves start slightly above the 50 per cent. level. The animals make some progress toward a mastery of the problem during the initial 100 trials. (2) Curves $A$ and $C$ exhibit no significant differences. The rate of learning is the same for the two intervals of 15 and 35 seconds. (3) All three curves are practically identical for the initial $\mathrm{x}, 000$ trials. Evidently the rate of progress for the initial stages of learning does not depend upon the time intervals employed. (4) Curve $B$ rises quite rapidly from the roooth to the 1200 th trial. This fact is the only evidence that the rate of learning may be a function of the length of interval. Other modes of explanation are possible. Unfortunately this group contained but three rats at the end of the experiment. The progress of any animal in this problem is highly erratic; a high record may be made consistently for a number of days, and this period may be followed by one in which extremely poor records are achieved. This sudden rise of the curve may be a chance spurt and the group might have been unable to maintain this level of excellence if the tests had been continued. The experiment should have been continued. Chance might still be a factor even if the group had been able to duplicate their records in succeeding trials. Individual success in such a problem is quite dependent upon chance factors. The experiment can hardly be regarded as a decisive test of the influence of the time interval upon the rate of learning. The evidence favors the assumption that the differences employed in the experiment were without effect.

2. A second experiment was conducted by the writer. Groups $A, B$, and $C$, each containing 6 rats, were tested for intervals of 5 , ro and 15 seconds respectively. Several conditions differed from those of the preceding experiment. The animals had had previous experience in a maze problem. Ten trials per day were given each animal for seven days of the week. But one rat was tested at a time. Owing to sickness no tests were given for a week at the end of the gooth trial. All control tests previously enumerated were given at the end of the experiment. 
The group records are represented by the graphs of Figure 3. Again the initial values are greater than 50 per cent. From an inspection of the curves it is evident that no group probably would have attained a proficiency of 90 per cent.

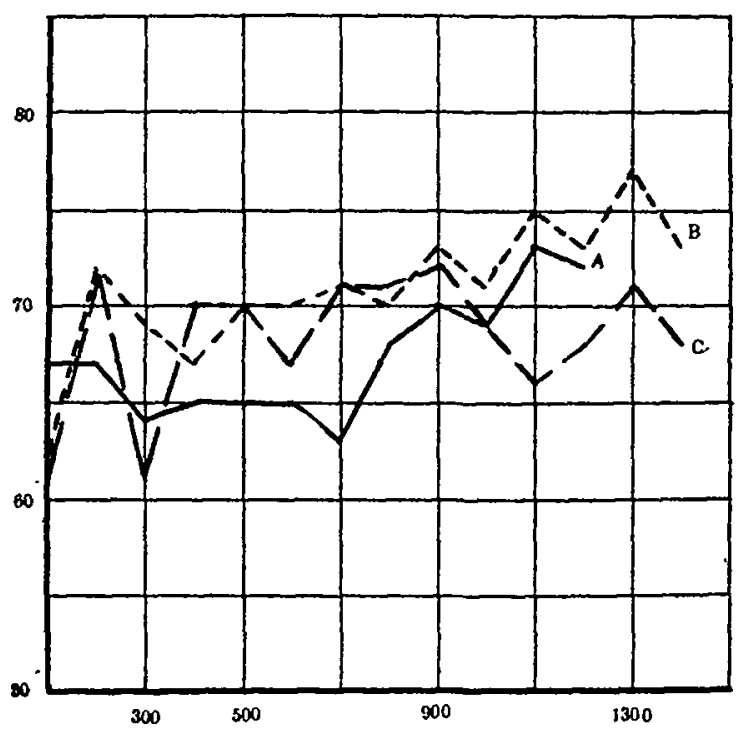

Ficure 3. The graphs $A, B$, and $C$ represent the average percentage of correct choices for successive 100 trials for the intervals of 5,10 and 15 seconds respectively.

within 3,000 trials. For this reason the experiment was discontinued. However, some rats did attain this level of efficiency during the experimentation. Other animals were making progress and gave every indication of final success. Some rats soon fell into fixed position habits and gave no indication of further progress. Group $B$ made the best records rather consistently. Their records are the highest in $I I$ of the 14 cases of comparison. Two rats of this group made scores of 90 per cent. of correct choices, while only one animal in each of the other groups attained this level of proficiency. There is but little difference between graphs $A$ and $C$. Group $C$ on the whole made the better initial records, while group $A$ made the more rapid progress in the later stages of the experiment. As to the influence of the 
time interval upon rate of mastery, the records indicate that ro seconds may be the most favorable of the three intervals.

3. A third experiment was conducted by Miss Koch. She tested group $A$, consisting of seven animals, for a rosecond interval, and group $B$ containing six rats for a 15second interval. The conditions of the experiment were the same as those of experiment 2 with the following exception. By means of the swinging door $Z$ (see Fig. I), the entrances into the paths $R$ and $L$ were alternately closed and the animals were thus forced to run in the given sequence of $R, L, R, L$, etc., for each day's series of trials. But one path was open at a time; errors were impossible; the rat was compelled to respond correctly in the desired sequence. To obtain records of the rat's progress in mastering this coördination, test series were interpolated every fifth day, in which the door $Z$ was removed and the animals were allowed to choose between the two paths as in the former experiments. The percentage values for each 100 total trials are thus based upon two test series of 10 trials each.

The scores for these two groups are represented by graphs

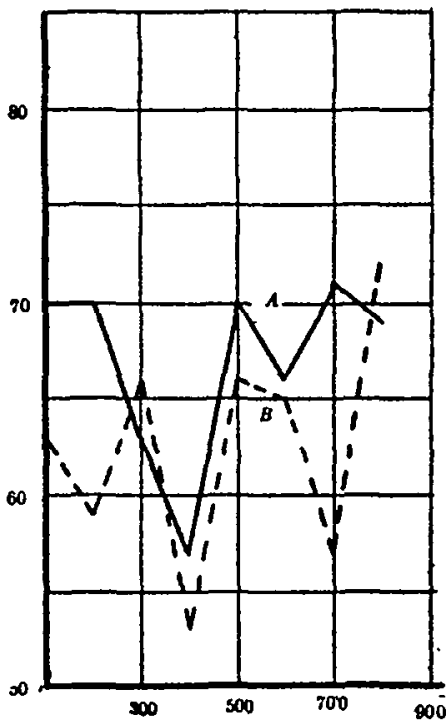

Ficure 4. The graphs $A$ and $B$ represent the average percentage of correct choices for successive 100 trials for the intervals of 10 and 15 seconds respectively. $A$ and $B$ of Figure 4. The initial records are quite high and neither group gave much indication of further progress. Evidently progress was confined mainly to the initial 100 trials. Certain animals succeeded in attaining a proficiency of 90 per cent. of correct choices. The graphs gave so little indication of an early group mastery that the experiment was discontinued. The high degree of irregularity of the successive scores is due to the small number of trials upon which 
the values are based. Group $A$ made the better record. Their scores were the higher in six of the eight comparisons. Three rats of this group made a record of 90 per cent. while but one rat of group $B$ attained this degree of proficiency. The group difference is probably not very significant, but the results indicate that 10 seconds is a more favorable interval than 15 seconds.

The character of the curves in experiment I is radically different from the type of curve obtained in experiments 2 and 3. The latter curves exhibit the greater progress for the first 200 trials, and maintain a higher level for 500 trials; From this point on the curves of experiment I rise much more rapidly and maintain this ascendency for the remainder of the experimentation. The explanation of this difference in rate of progress must be sought in the different conditions of the experiments. Length of interval is not a factor, as the I5 second interval was employed in all three experiments. The previous experience of the animals in experiment 2 may account for the greater initial progress of these groups, but this factor can hardly explain the poor records of these animals in the final stages of the experimentation. Moreover the rats used in experiment 3 lacked previous experience. The technique of the experimenters may have been a factor. The rats employed in experiment I may have learned to rely upon visual cues from the experimenter in making their choices, as this possibility was not eliminated by control tests. The number of trials per day seems the more probable cause, as 20 trials per day were given each rat in experiment $I$ as compared to to trials in experiments 2 and 3 . On this hypothesis 10 trials per day is the more effective distribution for the initial stages of mastery, while greater progress can be attained by the use of 20 trials after this early period of adjustment to the problem. Such a fact will constitute an exception to our present knowledge concerning the relative values of concentrated and distributed trials. This conception must be regarded as a tentative hypothesis to be tested by further experimentation.

Our experiment proves that animals can form an associa- 
tion between two terms separated by a time interval as large as 35 seconds, provided that this interval is filled in with some rather constant type of activity such as eating. There is no conclusive evidence that the rate of learning is a function of the length of time interval employed. Certainly the rate of learning does not vary directly with the length of interval. There was some indication in two experiments that ro seconds may be more favorable than either a 5- or 15 -second interval. In the first experiment the 25-second group mastered the problem more readily than did either the 15- or 35-second groups. All of these differential results may well have been due to chance factors. In the absence of any conclusive evidence to the contrary, one must assume from our data that the speed of forming the association was not dependent upon the size of the intervals used. One is not justified, however, in extending this conclusion to other intervals. One must also admit that the method of the experiment is not particularly well adapted to the solution of the problem.

This problem of the relation of the rate of learning to the time interval has received but little attention from experimenters in either the animal or human field. Bergstrom and Froeberg have made excellent studies with the use of human subjects. In Bergstrom's ${ }^{1}$ first experiment the subjects were required to memorize a list of 12 syllables which were visually presented in succession. Each syllable of the series was exposed to view for a period of $82 \sigma$, and a given time interval was allowed to elapse between the successive exposures of the syllables. The problem thus required the association of a series of 12 terms successively exposed when the series of exposures were separated by a given time interval. Three subjects were tested. Each memorized 12 lists of syllables per day for four days. The subjects were requested to pronounce silently each syllable but once at the time of its exposure. After four repetitions of a list of syllables, the amount memorized was measured by the number of errors

${ }^{1}$ Bergstrom, John A., 'Effect of Changes in the Time Variables in Memorizing, together with some Discussions of the Technique of Memory Experimentation,'Amer. J. of Psychol., 1907, 18, pp. 206-238. 
made in an immediate written reproduction. The intervals between exposures were $302 \sigma, 686 \sigma$ and $1454 \sigma$, and the average number of errors for the three intervals respectively were ro.3, 8.9, and 7.5. In a second test 30 subjects were used and the material consisted of lists of 10 words and lists of 10 letters. This material was presented orally at a rate controlled by the beating of a metronome. Since the time necessary to pronounce each term of a series presumably approximated a constant, the differences of rate will represent differences of time interval between successive exposures. The time intervals employed were $.5,1$, and 2 seconds. Each list was presented but once and the amount memorized was tested in terms of the errors made in an immediate written reproduction. The average percentages of error for the lists of words were $51.12,36.5$, and 23.9 for the .5 , I, and 2second intervals respectively. The corresponding percentage values for the lists of letters were $44.09,42.65$, and 38.44 . In both tests the amount learned increased as the interval was lengthened, and the author indicates his belief that this relation will obtain for much larger intervals than those employed in the experiment.

Froeberg's ${ }^{1}$ subjects memorized a series of five pairs of syllables. Each term of the pairs was exposed for about one fourth of a second. The members of the pairs were exposed in succession and the interval between their exposures was varied from 0 to 5 seconds. The interval between successive pairs was a constant. Each series was given two repetitions as a rule, and the amount memorized was tested ro seconds later. In the test for recall the stimulus syllables were presented in a new order and the subject was required to supply the missing member of each pair. The subjects were requested to inhibit the tendency to articulate the syllables and to refrain from all thought associations. The average percentages of correct responses for the group of seven subjects were $49,45,48,51,49$, and 49 for the intervals of $0,1,2,3$, 4 , and 5 seconds respectively. These figures indicate that the

1 Froeberg, 'Simultaneous versus Successive Association,' Psycroz. REv., 1918, 25, pp. 156-163. 
rate of learning is not a function of the length of interval. The experiment was repeated with a group of four subjects with one change of condition. Numerals were visually exposed during the interval of time between the exposures of the members of a pair which were to be associated, and the subjects were asked to perceive and read these interpolated numerals during the interval. This procedure was designed to prevent a memory survival of the first member of a pair during the interval separating it from its associated member. With these conditions the average percentages of recall for the above intervals were $45,34,36,40,24$, and 22 and the rate of learning decreased with the length of interval.

There are but two experiments on this topic in the field of animal psychology. Thorndike taught cats to climb up three feet on the wire netting constituting the sides of their cage in order to obtain food held in his hand. The cats had thus learned to associate the act of climbing to a given spot with the visual stimuli of food in the hands of the experimenter. Thorndike now sat at a distance of 8 feet from the cage, clapped his hands four times and said 'I must feed those cats.' After a lapse of Io seconds, he took food, walked over to the cage and fed them as usual. This procedure was repeated in order to determine if the animals could learn to associate the act of climbing with the visual and auditory stimuli involved in the clapping of the hands and repeating the phrase. Such an association was soon formed and the cats climbed up for the food as soon as the new stimulus was given without waiting for the experimenter to bring it in the usual manner. The animals thus learned to associate two terms which were at first experienced successively with an interval of 10 seconds between them. Thorndike did not vary this interval in order to determine the relation between its length and the rate of learning. The experiment merely proves that cats under these conditions can learn to associate two terms over an interval of ro seconds.

In a previous experiment with the alternation problem,

IThorndike, 'Animal Intelligence,' p. 11 r. The MacMillan Co. 
the writer ${ }^{1}$ employed an interval of 16.5 seconds, and a group of white rats mastered the problem with a high degree of proficiency. The length of the interval was not varied during the learning process. The experiment indicates again that rats can form an association over an interval of 16.5 seconds. The experiment was defective in that the apparatus permitted the maintenance during the interval of a motor attitude characteristic of the previous choice. It was thus possible that the two choices were based in part upon the two distinctive motor attitudes maintained during feeding. On this hypothesis the interval between stimulus and response would be much less than 16.5 seconds.

There are three possible types of explanation of the fact that association can bridge a considerable interval of time. (I) One may assume the memory survival of each term during the interval until the succeeding term is presented. The connection is thus established between two simultaneous activities - the perception of one term and a conscious representation of the preceding one. (2) The connection may be mediated by some third factor which is present during the interval. A series of syllables may be memorized by associating each with its position in a temporal sequence. Almost any kind of mental activity may serve such a mediating function. (3) The third possibility assumes that the two terms may be associated directly, that each activity becomes connected with the retained effects of the preceding activity. An inspection of the various statements concerning association in the texts and treatises on general psychology reveals the fact that simultaneous or successive presentation is regarded as an essential condition for the formation of an effective connection. The term 'immediate' is not explicitly defined. Apparently the authors imply one of two things: The two experiences must be in temporal contact with no interval or interpolated activity between them, or other experiences may intervene but the two terms to be connected must exist within the 'specious present.' On this basis only conscious 384 .

' Carr, Harvey, 'The Alternation Problem,' J. of Animal Beh., 7, pp. 365- 
activities can be associated, and our third possibility of connection is excluded.

Thorndike in his experiment with cats considered the first and third possibilities. He regarded a direct connection as highly improbable, and hence inferred that the association must have been mediated by a memory image of some sort. The experiment is thus presented as a proof of the existence of an ideational capacity in cats. The second possibility was ignored. Such a mediating factor may have been the visual perception of the experimenter. On this hypothesis the experience of the food became associated with the perception of the experimenter and this latter term was again associated with the hand clapping and the spoken words.

In Bergstrom's experiment memory connection was possible. Temporal association and other indirect thought connections may have been established. The character of the results indicates such modes of connection, for the longer the interval the greater is the possibility of a memory review and the development of effective thought connections.

In Froeberg's experiment temporal association was excluded by the method of paired associates. The temporal order in recall was different from that in presentation. In the first test the subjects were requested to refrain from developing thought connections and to inhibit the tendency to articulate the syllables. In spite of these instructions, the subjects reported that the association was mediated by memory. In the second test the subjects were required to read numerals during the interval. This activity was interpolated for the purpose of preventing any memory connection, and the introspective reports indicated that the procedure was successful. Froeberg thus concludes that associations can be established directly over an interval of at least 5 seconds, and that within these limits the rapidity of learning decreases with the length of interval.

There are three possible objections which can be urged against Froeberg's conclusions. (I) One may assume that all memory and thought connection was not wholly excluded. This hypothesis can not be regarded as wholly irrational as 
such modes of connection are many and devious, while some are by no means capable of being easily and readily observed. Such an objection will apply to practically every experiment which employs human subjects. (2) One may assume that the associations were mediated by these interpolated activities themselves. With this conception the subjects were memorizing a series of terms presented in immediate succession,-a syllable, a numeral, a second numeral, and the final syllable. Owing to the instructions for memorizing and recall only the final term is reproduced. This explanation is similar to that suggested for Thorndike's experiment, viz., that the connection may have been mediated by the visual perception of the experimenter. (3) One may admit that the connection between the syllables was formed directly but deny the validity of Froeberg's factual correlation between rate of learning and length of interval on the ground that the interpolated activity constitutes a distraction.

It is not our purpose to maintain the validity of the above objections. Rather we wish to emphasize the diffculties involved in securing a decisive proof of the possibility of a direct associative connection with the use of human subjects, and to suggest that animal experiments offer certain advantages for the solution of the problem. Presumably indirect thought connections can be effectively excluded by this means. Few will assert that our animals mastered the alternation problem by means of a concept of temporal order, viz., that they developed an idea of the principle of alternation and kept track of the proper response by counting the number of trials. Neither is the employment of other conceptual devices very likely. The experiment was devised in order to exclude a connection by means of the interpolated activity. The animals form two distinct associations while the interpolated activity of eating was the same in both cases. It was assumed that one interpolated activity could not mediate two distinct associations. Further reflection, however, has induced some scepticism of the truth of this proposition.

Granted the elimination of all indirect modes of connec- 
tion of the second type, one must choose in our experiment between the hypotheses of a memory survival or of a direct connection. As noted Thorndike regarded the former explanation as the more probable. The writer prefers the latter conception. On the one hand the memory hypothesis encounters many factual and theoretical difficulties which need not be enumerated at this time. Animals are preferable to human subjects in the attempt to avoid a memory connection. A direct connection, on the other hand, seems logically feasible on the basis of certain generally accepted explanatory principles.

Let us take the problem of associating the stimulus $X$ with a certain movement $Y$. Originally the stimulus $X$ will not evoke the act $Y$, and the problem consists of so connecting them that $Y$ will become the natural motor outlet of the nervous impulse aroused by $X$. This connection is usually secured by the simultaneous functioning of the two terms, and the experimenter achieves this result by evoking the act $Y$ by the use of some stimulus $B$ at the same time that stimulus $X$ is presented. The whole task of the experimenter consists of repeating this simultaneous presentation until the connection is effected; the rest of the process must be left to nature.

A usual type of explanation assumes that the nervous impulse aroused by $X$ is deflected from its normal channels over to the motor outlet $Y$ because of the lowered resistance or tension in that center due to its activity. This conception involves two assumptions: (I) The motor outlet of an incoming sensory impulse is in part a function of the relative resistance, tension, or susceptibility to response of the various motor centers. Each sensory impulse naturally tends to be drained off into that motor channel which for the time offers the minimum of resistance. (2) The conception assumes that the resistance or tension of any center is decreased by the functional activity of that center. The point which we wish to emphasize in this mode of explanation is the fact that the state of decreased resistance is the essential condition which mediates the desired connection. The induced ac- 
tivity in the motor center is not important per se, it is but a means to an end. Theoretically an experimenter can establish an associative nexus between the given factors by any device which will effect a decrease in the resistance of the motor center.

The conception of resistance is also frequently applied to the phenomena of retention and habit. The hypothesis assumes that the repeated activity of a center permanently lowers its resistance or increases its susceptibility to activity. By sufficient practice any center may be brought into a rather permanent state of a high degree of readiness for response. With such a center, activity produces the maximum of effect, and this effect rather rapidly diminishes with the cessation of activity until the relatively permanent level of habit is reached. On the basis of this conception, there are no a priori reasons why an effective association can not be established over a considerable interval of time, provided that one of the factors, e.g., the motor center $Y$, has been developed by practice to a state of readiness much greater than that of any other motor outlet. One should expect that simultaneous presentation will constitute the most favorable condition for the formation of an association but that the rate of learning will diminish as the time is increased up to a certain interval, after which further variations of interval will have but little effect.

Certain writers prefer to regard a state of activity in a center as a necessary condition of its lowered resistance or readiness for response. When the motor center $Y$ is evoked to activity by the controlling stimulus $A$, it is assumed that this activity will persist in a subconscious and subliminal form after the stimulus is removed. Applying this conception to habit, a nervous activity when once aroused never completely subsides. A center when once active never attains a state of functional quiescence. Association over a time interval is as feasible on this basis as on that sketched above. The writer sees no reason why readiness for response can not be conditioned by a state of functional quiescence as well as by a state of subliminal excitement. The additional hypo- 
thesis is thus gratuitous. While one can easily conceive of the persistence of an activity for a short interval of time on the principle of inertia, yet the further assumption of an indefinite continuance rather taxes one's credulity.

In conclusion, this paper advances the following propositions. An associative nexus can be established over a considerable interval of time. Our results do not permit of any statement that the rate of learning is a function of the intervals employed. With the possible exception of the interpolated activity, the connection was not mediated by some third factor. As an explanation one must choose between the hypotheses of direct connection or of memory survival. The choice must be determined largely by $a$ priori considerations. We do not assert the validity of the resistance conception as applied to the phenomena of habit, retention, and the formation of associations. We do note the fact that such a mode of conception is quite generally accepted, and we do maintain that on the basis of these assumptions the hypothesis of a direct connection is logically permissible. One must either admit the possibility or deny the validity of the resistance conception. 